\title{
Visualizing the Neurobiology of Trauma: \\ Design and evaluation of an eLearning module for continuing professional development of family physicians in the Online Psychiatric Education Network
}

\author{
Sarah Kim, Leila Lax, Dana Ross, Derek Ng, Diana Grossi, Renu Gupta, Sanjeev Sockalingam, \\ Robin Mason and Valerie Taylor
}

\begin{abstract}
Traumatic experiences can change brain structures and compromise emotional, cognitive, and bodily functions, thereby debilitating patients. Yet, trauma is not well understood by physicians and few educational resources are available, despite its prevalence. The goal of this design research project is to develop and evaluate $2 D$ animations in a case-based eLearning module. Complexities of post-traumatic stress disorder, including physical, emotional, and sexual abuse, are difficult to teach, talk about, and visually portray. Results of this study elucidate effective design dimensions of graphic narratives, keywords, and animations.
\end{abstract}

Keywords: medical animation, neurobiology, trauma, knowledge building, knowledge translation, design research

OPEN ACCESS

\section{Introduction}

\section{Post-Traumatic Stress Disorder}

Traumatic events, whether personally experienced or witnessed second-hand, can compromise emotional, cognitive, and bodily functions, leading to debilitating symptoms for patients (Heim et al., 2009; Sherin et al., 2011) and a diagnosis of post-traumatic stress disorder (PTSD) (Sareen 2014). Trauma can result from a one-time event, for example as a witness to violence, or it can occur over an extended period of time, as in the case of child abuse or intimate partner violence (Sareen 2014). When a significant stressor occurs, the hippocampus (involved in processing memory) and the amygdala (involved in processing emotions) are flooded with stress hormones (Heim et al., 2009). The individual is unable to process the traumatic experience as a finished event (even though there is no longer a threat), and it remains active in the brain (Taylor et al., 2015; Sherin et al., 2011). Thus, the symptoms of PTSD can surface many years after trauma, with delayed onset occurring in $25 \%$ of cases (Sareen 2014). As symptoms may be triggered at any time, physicians must have the knowledge and skills to identify and treat patients with care in order to avoid potentially retraumatizing the individual (Taylor et al., 2015; Sareen 2014).

PTSD is a global health issue and is categorized in the Diagnostic and Statistical Manual of Mental Disorders (DSM5) under "Trauma-and Stressor-Related Disorders"
(American Psychiatric Association 2013). It has a lifetime prevalence of $9.2 \%$ in Canada and $90 \%$ of these cases are comorbid with another physiological or psychological diagnosis (Van Ameringen et al., 2008; Sareen 2014). The National Comorbidity Survey-Replication found that the median time to first treatment of an individual with PTSD in America was approximately 12 years and only about $60 \%$ of affected individuals had received any treatment during their lives (Wang et al., 2005).

PTSD is believed to reflect real stress-induced changes in neurobiological systems, yet it is not well understood by family physicians. Few educational and community resources are available relevant to PTSD, despite its high prevalence (Gamm et al., 2010). Particularly lacking are educational tools focusing on neurobiological changes associated with PTSD.

\section{OPEN eLearning Module}

A preliminary needs survey of Ontario family physicians and community health care practitioners exposed the need for continuing education that included a better understanding of the neurobiology of trauma (National Physician Survey 2010). In Canada, the lack of access to specialized mental health and psychiatric care is a significant problem, causing patients to rely on their family physicians and community health care practitioners to address these often-complex issues. However, many may feel they lack the necessary knowledge and in-depth training to do so.

The Online Psychiatric Education Network (OPEN) is a webbased eLearning initiative for the continuing professional development (CPD) of family physicians and interprofessional health care practitioners. OPEN aims to provide evidence-based knowledge building, relying on the most up-to-date information for knowledge translation to practice. The goal is to ultimately increase community capacity and to improve patient and family care. OPEN is a collaboration between Biomedical Communications, Faculty of Medicine, University of Toronto and Women's College Hospital, Department of Psychiatry, conceptualized by Dr. Leila Lax and Dr. Valerie Taylor (Lax et al., 2015). All eLearning modules on OPEN use innovative visual media design strategies and offer efficient anytime, anywhere, any pace, internet-based education (Lax et al., 2015; Titov et al., 2002; Andrews et al., 2010). In 2012 The Mental Health Commission of Canada identified online education for CPD as a priority. 
The OPEN Neurobiology of Trauma module combines relevant medical content, compelling, didactic animations, and a friendly user interface design. A competency-based framework is employed for guided self-learning with opportunities for reflection and linking learning to practice. The purpose of this introductory module is to improve family physician understanding of the science underlying the neurophysiology of trauma. Additional chapters on clinical best practices and treatment are to be added to this module.

\section{Design Research Model}

This project benefits from the use of a design research model (Bereiter 2002; Lax et al., 2011) and the engagement of a committee of seven professionals of diverse expertise, who collaboratively provided comprehensive feedback to support a successful educational experience and outcome. All members hold a PhD and/or MD degree and have specialized backgrounds in psychiatry, biomedical communications, design research, education, CPD, and public health. Monthly meetings ensured timely progress and continuous formative feedback for iterative refinements. This co-design method (Lax 2015) strengthened the creative and evidence-based development of the module and was a crucial aspect of the design process.

\section{Methods}

\section{D Animations, Multimedia, and Intentional Design}

A paucity of CPD materials for family physicians on the topic of trauma and PTSD and a particular lack of visual resources has been identified (Gamm et al., 2010). Animations have been shown to be powerful teaching tools, particularly when incorporating dual pathways of both visual and audio elements in linear storytelling to encourage deeper learning (Mayer 2008; Mayer 2014). This design research project aims to demonstrate how multimedia can be a powerful teaching tool if designed effectively. Design dimensions will be examined along with knowledge improvement. In an effort to teach in innovative ways, the first author, Sarah Kim created a series of 2D animations in a contextualized trauma case. Graphic narratives of clinical case scenarios are typically designed from the physician's perspective. This module was designed from the patients' perspective, to give expression to the often-hidden thoughts and explicate the anxieties and feelings of the patient. In addition, keywords from the audio content were graphically visualized and animated to highlight important information and make it more memorable, according to multimedia design theory (Mayer 2008).

PTSD deals with complex, nuanced, and sensitive subject matter, including physical, emotional, and sexual abuse (Sareen 2014), that is difficult to teach, talk about, and visually portray. We believe the use of visual metaphors in a graphic narrative would tactfully convey such information without overwhelming the learning audience. We used accurate schematic visualizations rather than scientifically dense illustrations to avoid teaching the neurobiology components like a textbook. To maximize learner focus and knowledge building, key concepts were identified and emphasized while extraneous information was reduced (Mayer 2008).

The design research process of creating this module required the integration of scientific content, visual design and technology (Figure 1). A knowledge translation competency framework was created based on previous work by Lax and colleagues in 2011. All medical content was written by Dr. Dana Ross, a psychiatrist who specializes in psychological trauma. Content was strategically divided into three chapters, each focusing on one key concept. Dr. Ross wrote the animation scripts, the unfolding case narrative, the knowledge translation commentary, as well as the pre- and post-test questions.

These components provided the foundation on which the visual design was built by the lead author, Sarah Kim. Broadly, there were two major design areas: the first dealt with the creation of the case animations and the second dealt with the creation of the module website to support all eLearning program components, such as Pre-test/Post-test, competencies list, reflections-on-practice, resources, etc.

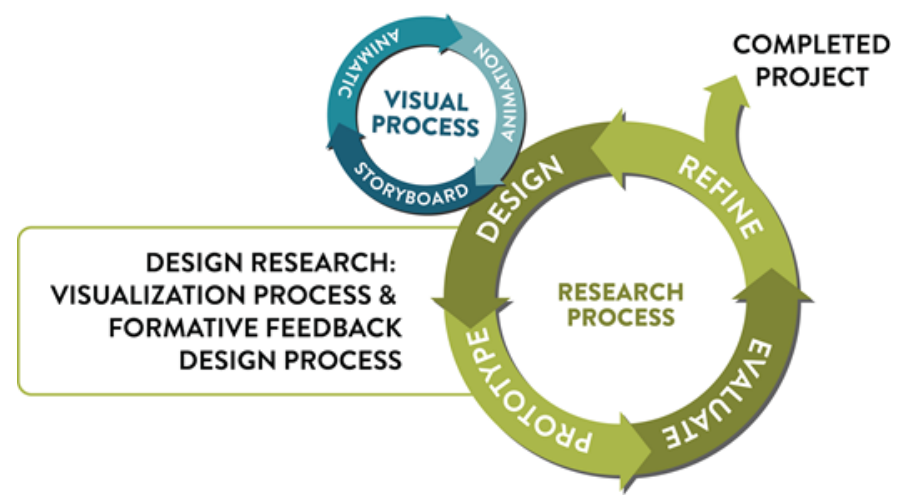

Figure 1. Design research is iterative and integrated; the process of visualization and development is conducted alongside the process of evaluation and formative feedback aimed at iterative improvement and refined design.

The first visual task the lead author faced was to draw storyboards to illuminate the scripts. These storyboards underwent multiple iterations, as different visualizations were explored around a range of traumatic events, such as sexual abuse and war trauma. The storyboards were critiqued by the committee, discussed, and refined to portray the most common scenarios in the most sensitive and meaningful way. Kim determined the character design and visual pace at the storyboarding stage. Once finalized, an animated storyboard was created to add motion, timing, transitions, and flow to the story. The final animations were then created on Adobe AfterEffects ${ }^{\circledR}$ and Illustrator ${ }^{\circledR}$ with full color and motion. 


\section{Design Intentionality}

Creation of this module presented numerous design challenges that required innovative design thinking and intentional problem solving. One challenge of this module was to communicate neuropsychiatric linkages between concepts of trauma, neurophysiology, and patient assessment and management. We used graphic metaphors, such as a moving ball for the amygdala, to simplify neuroanatomical information, yet retain accuracy and representational complexity in visual descriptions of the nature of PTSD. Nuances in the music or motion were also used to contribute to the overall mood and highlight key concepts important to understanding trauma.

We also wanted to render scientific information in a way that humanized and validated patient experiences; thus, there are more scenes in the animation including a character in a situation rather than diagrams and charts. Patient facial expression, body posture, and overall affect are examples of symptoms that are better communicated with illustrations than just text. The hope is that these subtle visuals that occur in a clinic setting will link such symptoms to the doctors' practices for knowledge translation. The animations also follow a single woman's journey for familiarity and continuity of care applicable to the family physician.

Another challenge was to deliver the animated information at a pace that was conducive to learning but also kept the viewer engaged. Since the target audience's medical knowledge is high, we simplified the complexity of visuals to focus viewer attention on larger concepts. Our visual design offers something different than the traditional CPD case-based learning modules that are often merely composed of text. Motion graphics were used, which is a technique where words and images are animated. Motion graphics are known for their catchy visual rhythm and smooth but quick presentation of information. This design strategy allows the eLearning module to be brief, while emphasizing key learning points - which is well suited to CPD for family physicians.

A lively color palette was used to express openness and the images were rendered loosely and organically. A classic serif or sans serif typeface was used to label academic points while hand lettering was used to highlight more implicit information (such as unseen symptoms) to induce a more personable feel to such words.

In summary, it is important to note that every design decision was intentionally made to increase module effectiveness. These design dimension assumptions were tested in this design research study.

\section{Wireframing and User Interface Design}

A secondary visual design and technical task was to create the module webpages (Figure 2) that were based on the OPEN educational framework (Lax et al., 2015). This required the creation of a functional wireframe for the site, designing an aesthetic interface, and programming it using HTML and CSS.
The OPEN educational framework was customized for the module and includes module competencies, a knowledge Pretest and Post-test to guide self-assessment, and a Knowledge Translation (KT) Commentary, which is a comprehensive summary that connects the three chapters with additional neurobiological information and concepts for management of patients with PTSD. Online references and resources are made available and easily downloadable to encourage further independent learning. Lastly, a Reflection-on-Practice section is included to encourage reflection on knowledge building at the conclusion of the module and 12-weeks post-course to facilitate KT to practice (College of Family Physicians of Canada 2015; Hattie et al., 2014; Lax et al., 2015).

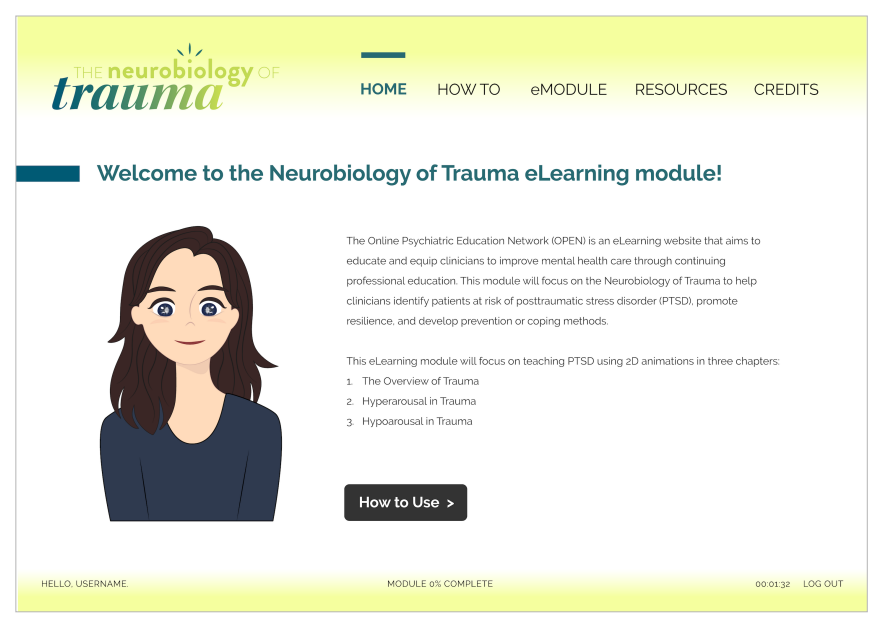

Figure 2. This screenshot shows the final webpage for the learning module. The functional wireframe and the visual designs were brought together to create the final webpage.

\section{Design Research Questions}

This design research study explored the following research questions: Is the eLearning design of this CPD module effective in improving physician knowledge of the neurobiology of trauma and PTSD specifically, and is the scientific information rendered clearly in the animations? Are the medical explanations conveyed accurately within the patient scenarios, and is the usability design of the website interactively seamless?

\section{Design Research Methods}

The University of Toronto Health Sciences Research Ethics Board approved this design research study (Bereiter 2003; Collin et al., 2004). A mixed methods case study approach was used to obtain quantitative and qualitative feedback to inform design and development. Design research aims to produce sustainable innovations through constructive formative feedback, collaboration, and multiple iterations for a more robust design (Bereiter 2002; Collins et al., 2004).

\section{Participants}

Six physicians (4 family doctors, 1 psychiatrist, and 1 psychiatric resident) who are currently practicing in Toronto, Canada, were recruited to participate in this online study from May 17 to May 30, 2016. Participants consented to completing 
a trauma knowledge Pre-test and Post-test and to providing survey feedback while reviewing the module on their own computers.

\section{Evaluation Instruments}

The Neurobiology of Trauma Pre-test and Post-test was administered to determine knowledge improvement. The Pretest was composed of 10 multiple-choice questions that were randomized on Post-test. No answers were provided at the end of the Pre-test, only on Post-test. Questions were based on content from all the three animations and were related to module competencies.

Lax's eLearning Design and Usability Survey (2011) was modified and employed in the evaluation of this module. The Neurobiology of Trauma eLearning Design and Usability Survey was composed of 111 Likert scale questions and 11 open-ended questions to obtain feedback on content accuracy, information clarity, usability, educational design, and multimedia aspects of the module, examined by chapter.

\section{Data Collection and Analysis}

The Neurobiology of Trauma module contains three chapters of an unfolding case scenario. Chapters 1 and 3 were completed for this design research study and only medical content text (no animation) was made available for review of chapter 2. The module website pages were also included for design feedback review.

The Neurobiology of Trauma Pre-test and Post-test were conducted within the module. Data was automatically collected online and then downloaded to Microsoft Excel for scoring by individual and in aggregate. Participants spent approximately one hour reviewing the module and simultaneously completing the eLearning Design and Usability Survey in Microsoft Word. The five-point Likert scale responses were transferred to Excel and analyzed using descriptive statistics. Qualitative feedback comments were transferred to Word, thematically categorized, and interpretively analyzed by two committee members.

\section{Iterative Design}

Both quantitative and qualitative feedback was used to guide iterative design refinements of chapters 1 and 3, completion of chapter 2, and educational design of the website. In particular, users were able to give clear feedback on audio and visual components of the module (such as pacing of the narration), which were used to develop a second iteration of the module.

\section{Results}

\section{Neurobiology of Trauma Pre-test \& Post-test}

Six participants completed the Neurobiology of Trauma Pretest and Post-test online, enabling individual matched analysis and in aggregate results. Overall, the average test score on Pre-test was $47 / 60(78 \%)$ and $53 / 60(88 \%)$ on Post-test demonstrating an increase of $10 \%$ (Table 1). Five of six participants showed an increase in knowledge from Pre-test to Post-test and one participant decreased in total score.

Content validity results were helpful in identifying strengths and weakness of questions. Three questions (Q1, Q2, Q6) showed the same results on Pre-test and Post-test. Two of those three (Q2, Q6) demonstrated perfect scores on both. Of the remaining seven questions, five showed knowledge improvement (Q5, Q7, Q8, Q9, Q10), while two demonstrated a decrease in score $(\mathrm{Q} 3, \mathrm{Q} 4)$. Both of these questions (Q3, Q4) pertained to the amygdala and its roles in PTSD. Module content and test questions were reviewed with the content expert; although information on the amygdala was framed differently in the hyper- and hypoarousal chapters, it was consistent. On review of the questions, it was found that the wording could be interpreted ambiguously and therefore it was modified for the next iteration.

\section{Neurobiology of Trauma eLearning Design \& Usability Survey Results}

The Neurobiology of Trauma eLearning Design \& Usability Survey was used to collect participant Likert scale quantitative and qualitative responses for all sections of the module, except chapter 2. Participants $(n=6)$ reviewed the module online and responded to the survey. Responses were tabulated and analyzed by the designer and committee to inform iterative and ongoing design of the eLearning module and animations. A committee formative feedback session was also conducted using the same survey questions. There were many areas of overlap, validating multi-perspective feedback and results.

\section{Quantitative Design Research Results}

Likert scale formative feedback responses to dimensions of eLearning design, content, and animation style were obtained and are shown in Table 2. Of particular note is the identification of the rapid pace of narration (Q13) that was to be addressed on the next iteration. All participants agreed/strongly agreed that the medical graphics were an effective way to convey information (Q20, Q63), that the hand-lettered words highlighted key points (Q21, Q64), that the use of graphic metaphors were effective in conveying concepts about the autonomic nervous system (Q68), that the simplified anatomical illustrations were effective (Q69), and that they would not prefer more detailed illustrations (Q70).

The majority of participants indicated that all scientific information was relevant to their learning and practice (Q33, Q34, Q46, Q47, Q81, Q82) and that that they think this module has help them to identify PTSD symptoms in their patients (Q105). However, participants indicated that more information is required on PTSD treatment and management (Q108). All participants indicated that they believe that their understanding of the neurobiology of PTSD improved as a result of reviewing this module (Q106). 
Qualitative Design Research Results

Qualitative survey comments were thematically categorized and collaboratively analyzed. Results focused on emergent categories: (1) quick pace of narration, (2) brisk rhythm of the animations, and (3) schematic representation of medical and neuroanatomical aspects and clinical situations. Qualitative analysis results were cross-checked with quantitative survey results to inform the iterative design of chapters 1 and 3 and development of chapter 2 .

In addition, reflective open-ended questions at the end of the survey provided many informative comments from a knowledge building and translation to practice perspective. Detailed discussion on validation of initial design propositions and changes to be made for the final iteration are presented in the next section.

Table 1. Matched Pre-/Post-test Analysis Respondents 6/6 (100\%)

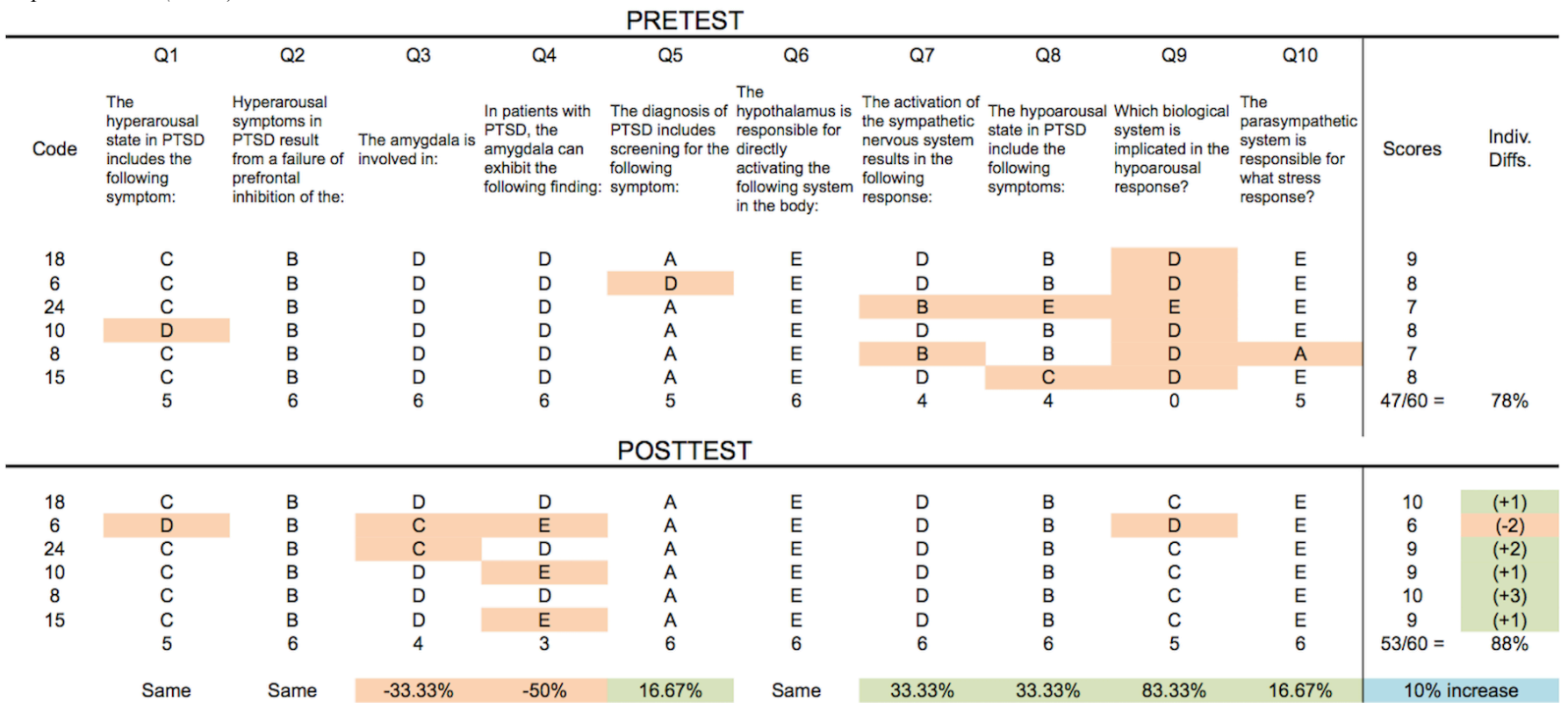

Note: Rows indicate participant's answers with summed score in the right column; columns indicate questions with summed scores at the bottom. Orange boxes indicate wrong answers.

Table 2. Neurobiology of Trauma eLearning Design \& Usability Survey Results Respondents 6/6 (100\%)

*Note: $N R=$ No Response; Likert scale $=$ Strongly Disagree to Strongly Agree

\begin{tabular}{|c|c|c|c|c|c|c|}
\hline Question & NR & SD & $\mathrm{D}$ & $\mathrm{N}$ & A & SA \\
\hline \multicolumn{7}{|c|}{ Homepage \& Introductory Pages } \\
\hline 1. The homepage helped me understand the organization of the program. & & & & 2 & 1 & 2 \\
\hline 2. The homepage made me interested in completing the eLearning module. & & & & 3 & 1 & 2 \\
\hline 3. At glance, the homepage helped me to understand the program navigation. & & & & & 4 & 2 \\
\hline 4. The How to Use page was informative & & & & 2 & 3 & 1 \\
\hline 5. The How to Use page was clear. & & & & & 4 & 2 \\
\hline \multicolumn{7}{|c|}{ Chapter 1} \\
\hline 6. The opening narrative of Ms. A was informative. & & & & & 2 & 4 \\
\hline 7. The narrative of Ms. A helped me understand the context for the animation. & & & & & 2 & 4 \\
\hline 8. The clinical narrative was realistic & & & & & & 6 \\
\hline 9. The amount of text on screen was appropriate. & & & & & 2 & 4 \\
\hline 10. The animation was aesthetically pleasing. & & & & & 2 & 4 \\
\hline 11. Information was presented as a good pace. & & & 1 & 1 & 3 & 1 \\
\hline 12. The animation visual graphics were well-paced. & & & & 1 & 2 & 3 \\
\hline 13. The narration was well-paced. & & 3 & & 3 & & \\
\hline 14. The narration was clear. & & & 1 & 1 & 1 & 3 \\
\hline
\end{tabular}




\begin{tabular}{|c|c|c|c|c|c|c|}
\hline 15. I would prefer a male narrator over a female narrator. & & 2 & & 3 & & 1 \\
\hline 16. The music was distracting. & & 1 & 2 & 2 & & 1 \\
\hline 17. I prefer the animation with the background music track. & & 1 & 1 & 2 & & 2 \\
\hline 18. I liked the style of the quick icons used in this animation (e.g. the list of functions). & & & & 3 & 2 & 1 \\
\hline 19. The motion in this animation was enjoyable to watch. & & & & 2 & 2 & 2 \\
\hline 20. The medical graphics (e.g. neuroanatomy of the brain) were an effective way to convey information. & & & & & 3 & 3 \\
\hline 21. The hand-lettered words effectively highlighted information. & & & 1 & & 3 & 2 \\
\hline 22. I paused the animation at least once to review a section. & & 3 & 1 & & 2 & \\
\hline 23. I found myself navigating through the animation a lot (e.g. rewinding to review, pausing to reflect). & & 2 & 1 & & 2 & 1 \\
\hline 24. The bedroom portrayal of "childhood trauma" was effective. & & & 1 & 1 & 2 & 2 \\
\hline 25. The movie theater portrayal of trauma trigger was effective. & & & & 4 & 1 & 1 \\
\hline 26. The character's facial expressions were appropriate. & & & & & 5 & 1 \\
\hline 27. The use of graphic metaphors (e.g. the animated amygdala) was effective. & 1 & & & 1 & 3 & 1 \\
\hline 28. The use of graphic metaphors helps me understand PTSD better. & 1 & & & 1 & 1 & 3 \\
\hline 29. There was a good amount of scientific information in this module. & & & & 1 & 1 & 4 \\
\hline 30. The visual presentation of the Chapter 1 webpage was aesthetically pleasing. & & & & 1 & 2 & 3 \\
\hline 31. Chapter 1 was interesting. & & & & 1 & 3 & 2 \\
\hline 32. Chapter 1 was informative. & & & & 1 & 3 & 2 \\
\hline 33. Chapter 1 was relevant to my learning. & & & 1 & 1 & 2 & 2 \\
\hline 34. Chapter 1 was relevant to my practice. & & & 1 & 1 & 2 & 2 \\
\hline 35. Chapter 1 was engaging. & & & & 2 & 2 & 2 \\
\hline 36. Chapter 1 was presented new information that I hadn't known before. & & & 1 & & 2 & 3 \\
\hline
\end{tabular}

\begin{tabular}{|c|c|}
\hline & Chapter 2 \\
\hline 37. & The narrative of Ms. A was informative. \\
\hline 38. & The narrative of Ms. A helped me understand the context for the future animation. \\
\hline 39. & The clinical narrative was realistic. \\
\hline 40. & The amount of narrative text on screen was appropriate. \\
\hline 41. & The scientific information was interesting. \\
\hline 42. & Chapter 2 was easy to navigate. \\
\hline 43. & Chapter 2 was well organized. \\
\hline 44. & Chapter 2 was interesting. \\
\hline 45. & Chapter 2 was informative. \\
\hline 46. & Chapter 2 was relevant to my learning. \\
\hline 47. & Chapter 2 was relevant to my practice. \\
\hline 48. & Chapter 2 presented new information that I hadn't known before. \\
\hline
\end{tabular}

\begin{tabular}{|l|l|l|l|l|l|}
\hline & & & & 3 & 3 \\
\hline & & & & & 6 \\
\hline & & & 1 & 3 & 2 \\
\hline 1 & & & 1 & 1 & 3 \\
\hline 1 & & & 1 & 1 & 3 \\
\hline 1 & & & 1 & 2 & 2 \\
\hline 1 & & 1 & & 3 & 2 \\
\hline 1 & & 1 & & 1 & 3 \\
\hline 1 & & & & 1 & 3 \\
\hline 1 & & & 1 & 3 & 1 \\
\hline
\end{tabular}

49. The opening narrative of Ms. A was informative.

50. The narrative of Ms. A helped me understand the context for the animation.

51. The clinical narrative was realistic

52. The amount of text on screen was appropriate.

53. Information was presented as a good pace.

54. The animation was aesthetically pleasing.

55. The animation was well-paced.

56. The narration was well-paced.

57. The narration was clear.

58. I would prefer a male narrator over a female narrator.

59. The music was distracting.

Chapter 3 


\begin{tabular}{|c|c|c|c|c|c|c|c|}
\hline 60. & I prefer the animation with the background music track. & & 1 & 2 & 1 & 1 & 1 \\
\hline 61. & I liked the style of the quick icons used in this animation (e.g. the list of functions). & & & & 2 & 1 & 2 \\
\hline 62. & The motion in this animation was enjoyable to watch. & & & & 1 & 2 & 3 \\
\hline 63. & The medical graphics were an effective way to convey information. & & & & & 2 & 4 \\
\hline 64. & The hand-lettered words effectively highlighted information. & 1 & & & & 3 & 2 \\
\hline 65. & I paused the animation at least once to review a section. & 1 & 3 & & & 2 & \\
\hline 66. & I found myself navigating through the animation a lot (e.g. rewinding to review, pausing to reflect). & 1 & 3 & 1 & & 1 & \\
\hline 67. & The bedroom portrayal of "childhood trauma" was effective. & & & & 1 & 4 & 1 \\
\hline 68. & The use of balancing portrayal of the autonomic nervous system was effective. & & & & & 3 & 3 \\
\hline 69. & The simplified anatomical illustrations were effective. & & & & & 3 & 3 \\
\hline 70. & I would have preferred more detailed anatomical illustrations. & & 1 & 5 & & & \\
\hline 71. & The character's facial expressions were appropriate. & & & & 2 & 2 & 2 \\
\hline 72. & The use of color in portraying information (e.g. light blue for numbness) was effective. & & & & 3 & 1 & 2 \\
\hline 73. & The use of graphic metaphors was effective. & & & & 1 & 3 & 2 \\
\hline 74. & The use of graphic metaphors helps me understand PTSD better. & & & & 2 & 2 & 2 \\
\hline 75. & There was a good amount of scientific information this module. & 1 & & & & 3 & 2 \\
\hline 76. & The visual presentation of the Chapter 2 webpage was aesthetically pleasing. & 1 & & & 1 & 1 & 3 \\
\hline 77. & Chapter 3 was easy to navigate. & 1 & & & & 2 & 3 \\
\hline 78. & Chapter 3 was well organized. & 1 & & & & 2 & 3 \\
\hline 79. & Chapter 3 was interesting. & 1 & & & 1 & 2 & 2 \\
\hline 80. & Chapter 3 was informative. & 1 & & & & 3 & 2 \\
\hline 81. & Chapter 3 was relevant to my learning. & 1 & & 1 & & 1 & 3 \\
\hline 82. & Chapter 3 was relevant to my practice. & 1 & & 1 & & 1 & 3 \\
\hline 83. & Chapter 3 was engaging. & 1 & & & 1 & 2 & 2 \\
\hline 84. & Chapter 3 presented new information that I hadn't known before. & 1 & & & 1 & 2 & 2 \\
\hline \multicolumn{8}{|c|}{ KT Commentary, $P$ and Posttest, Resources } \\
\hline 85. & The KT Commentary provided a good summary. & & & & 1 & 3 & 2 \\
\hline 86. & The KT Commentary was helpful to my learning. & & & & 2 & 3 & 1 \\
\hline 87. & The KT Commentary should include still or motion graphics. & & & & 3 & & 3 \\
\hline 88. & The Pretest and Posttest questions were clear. & & & & 3 & 1 & 2 \\
\hline 89. & The Pretest and Posttest questions helped guide my learning in the module even without the answers. & & & 1 & & 2 & 3 \\
\hline 90. & The Pretest and Posttest questions helped me understand my strengths and weaknesses. & & & & 1 & 2 & 3 \\
\hline 91. & I downloaded at least one resources/reference from this module. & & 5 & & & 1 & \\
\hline 92. & The references and resources collection at the end of the module was well organized. & & 1 & & 3 & 2 & \\
\hline 93. & The references and resources collection was useful for my learning. & & 1 & 1 & 3 & 1 & \\
\hline \multicolumn{8}{|c|}{ Overall (Usability and eLearning Design) } \\
\hline 94. & Navigation throughout this module was easy to use. & 1 & & & & 1 & 4 \\
\hline 95. & Access to all animation in the module was straightforward. & 1 & & & & & 5 \\
\hline 96. & Overall usability design was transparent and easy to use. & 1 & & & & 1 & 4 \\
\hline 98. & The eModule was easy to navigate. & 1 & & & & 1 & 4 \\
\hline 99. & The eModule was well organized. & 1 & & & & 1 & 4 \\
\hline 100. & The unfolding narrative of Ms. A was helpful to my learning. & 1 & & & & 1 & 4 \\
\hline 101. & This module was relevant to my medical practice. & 1 & & 1 & & & 4 \\
\hline 102. & I think working through this eLearning module helped me gain new knowledge about PTSD. & 1 & & & & 3 & 2 \\
\hline 103. & I think working through this eLearning module helped to understand the neurobiology of PTSD. & 1 & & & & 2 & 3 \\
\hline 104. & I think this module will help me communicate better with my patients about PTSD. & 1 & 1 & & 1 & 1 & 2 \\
\hline 105. & I think this module will help me identify PTSD symptoms in my patients. & 1 & & & & 4 & 1 \\
\hline
\end{tabular}




\begin{tabular}{|c|c|c|c|c|c|c|}
\hline 106. & I believe my understanding of the neurobiology of PTSD improved as a result of reviewing this module. & & & & 4 & 2 \\
\hline 107. & I believe my understanding of PTSD symptoms in patients improved as a result of reviewing this module. & & & 1 & 3 & 2 \\
\hline 108. & $\begin{array}{l}\text { I believe my understanding of providing balanced mental and physical health care improved as a result of } \\
\text { finishing this module. }\end{array}$ & 1 & & 2 & 2 & 1 \\
\hline 109. & I believe working through this eLearning module was a valuable experience. & & 1 & & 2 & 3 \\
\hline \multirow[t]{2}{*}{110.} & Based on my experience, I would recommend this module to a colleague. & & 1 & 2 & & 3 \\
\hline & & NG & $\mathrm{S}$ & $\mathrm{G}$ & VG & EX \\
\hline 111. & Overall, I would rate this module as & & 1 & 1 & 3 & 1 \\
\hline
\end{tabular}

*Note: $N G=$ Not Good, $S=$ Satisfactory, $G=$ Good, $V G=$ Very Good, EX=Excellent

\section{Discussion}

The OPEN Neurobiology of Trauma eLearning module combines science with technology and design to provide a visually innovative CPD experience for family physicians. The co-design method allowed the doctors, educators, and designers on the research committee to collaborate and improve ideas throughout the process. The formal research study then provided valuable insight from experts in education and psychiatry. All study feedback results were reviewed and deliberated upon for iterative design interpretation by the designer and committee; consequent changes were made to strengthen the module.

In summary, the Neurobiology of Trauma eLearning module was shown to improve knowledge. Pre/Post-test results demonstrated an overall increase of $10 \%$ from Pre-test $(78 \%)$ to Post-test $(88 \%)$ knowledge. Matched individual score improvements show that $9 / 10$ participants gained new (and accurate) information about the neurobiology of trauma. Overall eLearning Design and Usability Survey results demonstrated that all participants believed that their understanding of the neurobiology of PTSD improved as a result of this module, and the module was highly rated.

Positive formative feedback from the detailed Survey affirmed many module design choices, while negative and mixed feedback prompted opportunities to reconsider specific design dimensions and to improve the animations. Feedback was used to refine animations $\# 1$ and \#3 and to further develop animation $\# 2$.

Figures 3-5. These screenshots show Ms. A, the protagonist of the module. Many traumatic situations are repetitive in nature and often begin in childhood. These stories depict Ms. A's trauma as repetitive, ongoing, and domestic in nature since she was a child.

\section{Clinical Case Narrative Contextualization}

Clinical case narratives are provided at the beginning of every chapter and are text-based scenarios that unfold over the course of Ms. A's three visits to her doctor. Participants consistently rated the opening narrative of Ms. A as informative and helpful in understanding the context of the subsequent animation; $100 \%$ of participants also strongly agreed that the clinical narrative was realistic. These narratives contextualize the medical information, provide information on patient assessment and management, and add a humanizing feature to PTSD symptoms and the clinical scenario. Clinical case narrative text is foundational to case-based learning in medical education; combinations and seamless transitions between text and media, as designed in this study, will be used in other OPEN modules.

\section{Graphic Narrative Design of Images and Keywords}

Creative graphic design strategies were successfully implemented in this eLearning module. The sensitive nature of the subject matter demanded new conceptualizations to visually communicate information about trauma. Graphic narratives were developed to convey information on a patient's trauma history and current mental health situation (Figures 3-5). The multimedia images of the patient in these scenarios provide a rich understanding well beyond a textbased scenario and more comfortable than filmed acting. Survey feedback from all participants favoured this medical graphic narrative approach.

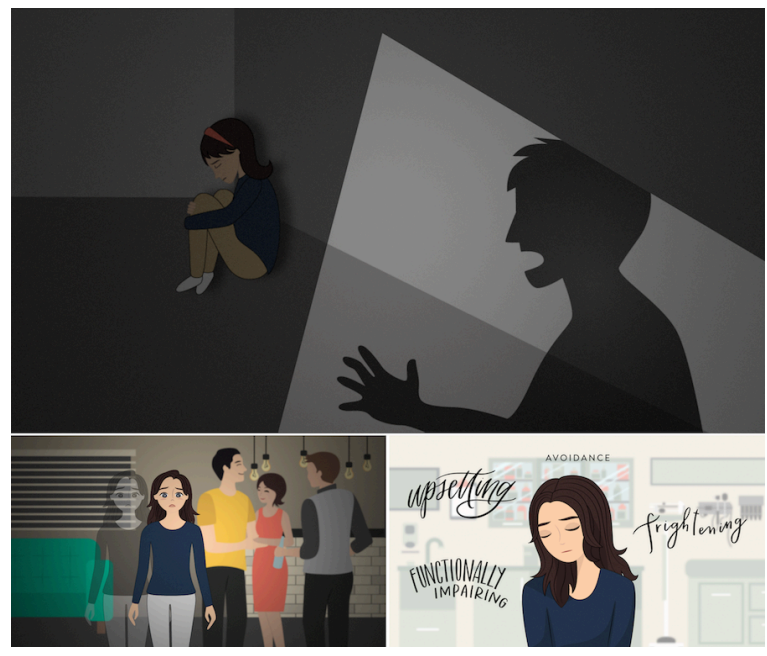


The technique of hand-lettering keywords also emphasized important concepts in the audio narration. Words were represented using expressive graphics inspired by its meaning. For example, the word "anxiety" was drawn with thin, wispy strokes and subtly animated to be shivering. This dual coding feature makes information more memorable.

\section{Animation Rhythm and Pace of Narration}

Qualitative and quantitative feedback from the design research survey regarding the rhythm of the animation and the pace of narration indicated that both were too brisk. Four of six participants indicated that the animations were too fast and no participants agreed that the narration was well-paced. The original intent was to create an eLearning module that was brisk, rhythmic and captivating in both visual and audio modes. Brisk rhythm was an important design feature intended to contrast with more traditional didactic modules. Traditional education tools are often used to teach textbook material and tend to be long in length and slow in presentation; however, the Neurobiology of Trauma eLearning module aimed to provide clinical information, such as patient affect, that are beyond the scope of a textbook. Content was created to be succinct. The goal was not to teach an anatomy lesson, but rather to build-on doctors' prior neuroanatomical and clinical knowledge to advance their understanding of PTSD to improve patient care. Each animation was created to be 2-3 minutes in length to maintain viewer's attention and to reinforce one main point per animation to focus physician learning.

Animations were reviewed and specific segments were modulated to decrease pace; however, it was decided that an overall brisk rhythm would be maintained. Importantly, the usability design of all animations provides full user control and the intent is that each animation be viewed multiple times. The balance between brevity and complexity of each animation scaffolds ease of usability for recursive knowledge building (Lax et al., 2009). We know that because of the nature and the time constraints of this design research study the animations were not replayed (as indicated in the survey), as per the original intent.

Modulation of pace and rhythm presented iterative design challenges. A design decision was made to insert more pauses in the narration and motion graphics in targeted areas, to slow the flow of information as needed, but to maintain the overall rhythm and preserve the original positive effects. For example, when functions or symptoms are listed in the animation, one extra second of time was added in between the words and longer pauses were added between certain sentences, especially following an important concept. The pause allows the graphics to remain idle on the screen a little longer to better enable information processing of complex concepts before transitioning into the next idea. These subtle design modifications were incorporated in all three animations.

\section{Schematic Scientific Representations}

Participants were asked if the schematic anatomical illustrations were effective for animations 1 and 3; half of the participants agreed and the other half strongly agreed (Q89). All participants indicated that they did not prefer more anatomical detail in the graphics (Q90). This confirmed the original design research premise of creating an eLearning tool with accurate, schematic illustrations to maximize attention and engage the audience in learning important concepts for knowledge translation, rather than focusing on details of realistically rendered neuroanatomy (Figures 6-9).

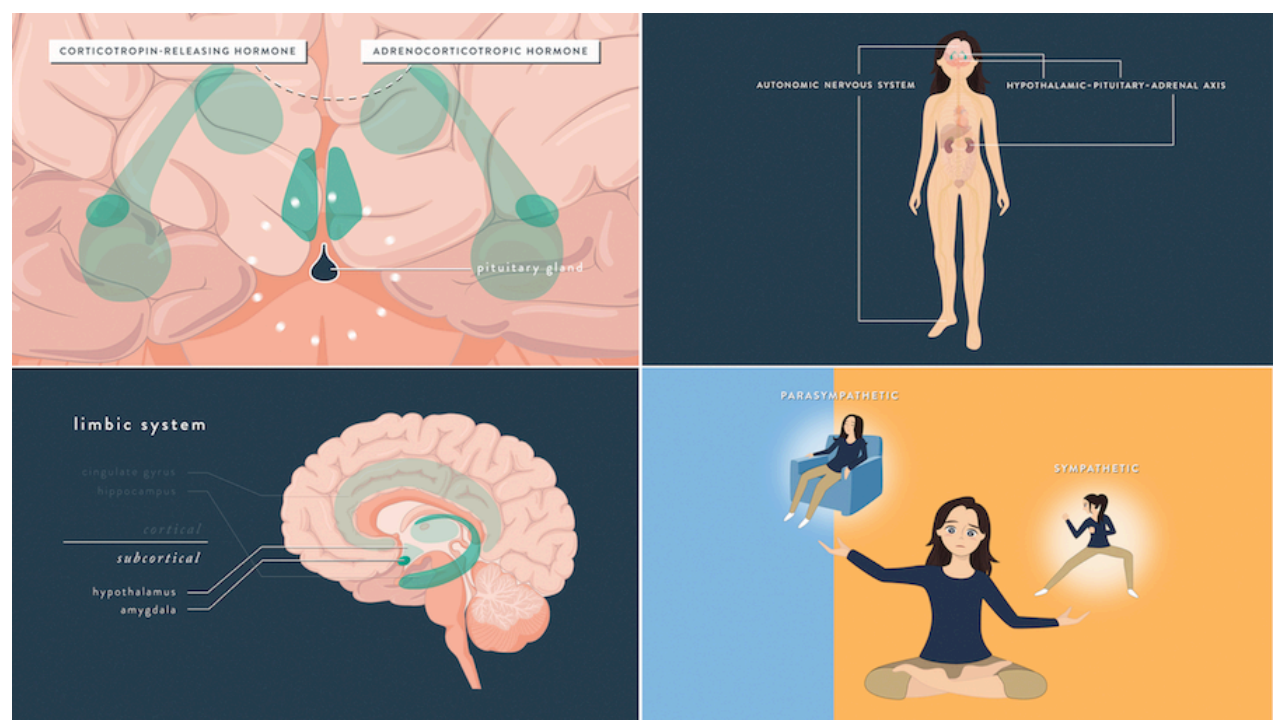

Figures 6-9. These screenshots show graphic renditions of neuroanatomy and metaphor conceptualizations. Hormones are depicted as balls and brain structures are simple overlain colors. Structures are isolated or labeled to show their significance and context. Color and metaphors are used to suggest concepts such as parasympathetic and sympathetic nervous systems. 
These results were crucial in developing animation 2, which contains the most amount of scientific information, e.g. explanations of neural pathways. All depictions of neurophysiology were rendered as simplified, yet scientifically accurate graphic representations that proved to be strong and well-understood visual metaphors. For example, rather than using chemical or molecular structures to represent hormones, different colored circles were used to show their emission. The graphic representation of the brain was drawn to emphasize key structures and their associations to contextualize relationships; details were reduced to metaphors. These graphic metaphors effectively highlighted key ideas for knowledge building. They also allowed for quick and easy rendering in production.

\section{Future Directions}

The Online Psychiatric Education Network aims to improve clinical practices for psychiatric care in the primary care setting by educating family physicians. It is important to note that an improvement in overall clinical practice and real-world outcomes requires the collaborative care of a variety of health care providers and health care systems (APA and APM, 2016). While this introductory module is an effort to engage family physicians on PTSD, it is limited in its scope and will benefit from additional resources. It is intended that future trauma modules focusing on clinical management, treatment options, and patient outcomes will augment this module. The design research and development of the Neurobiology of Trauma eLearning module, as discussed in this paper, serves as a prototype for visual, case-based learning. Results of this study will be used to drive the creation of additional chapters and future modules.

Upon accreditation and implementation of the Neurobiology of Trauma module as a CPD course, phase 2 of this design research study will be conducted with a larger sample of family physicians to measure the efficacy of this eLearning tool. Included in the CPD implementation will be an end-of-course reflective exercise and a 12-week post-course reflection-onpractice plan designed to scaffold knowledge translation to help physicians monitor improvements in their practices and note barriers to patient care. In summary, based on participant feedback in this study, this module should expand on the current Knowledge Translation Commentary to provide more comprehensive knowledge and information on patient treatment and management for further physician self-learning. Point-ofpractice resources could also be created and embedded in each chapter for physicians to download for ongoing use in practice. Lastly, it would be interesting to conduct a future study with a group of individuals affected with PTSD and to involve those individuals and patients in our design research process.

The Neurobiology of Trauma module will be offered online as a CPD course for family physicians through the University of Toronto, Faculty of Medicine, Office of Continuing Professional Development, in partnership with Women's College Hospital, Department of Psychiatry, Toronto, Canada.

\section{Conclusions}

The design of the OPEN Neurobiology of Trauma eLearning module goes beyond traditional text-based methods of teaching and learning about PTSD. The evidence-based medical visualizations and graphic narratives in this module clearly and accurately elucidate the scientific basis of PTSD. This module was designed to emphasize the patient perspective and has been shown to enable and improve physician understanding of the nuances and complexities of trauma, to support knowledge building, and to facilitate translation to practice.

In conclusion, innovative visual design strategies were created, tested and validated in this design research study, specifically motion graphics for keywords, schematically styled neuroanatomical renderings, graphic metaphors for visual complexity, and variations in rhythm/pacing of animations appropriate for recursive learning. Notable is the successful collaborative design research process used throughout the development and evaluation of the OPEN Neurobiology of Trauma eLearning module.

\section{Acknowledgments}

The OPEN Neurobiology of Trauma design research project was funded by Women's College Hospital. Programming of assessment components was funded by the Royal Bank of Canada and realized by Axon Interactive Inc. (Tabby and Jeff Rose). In-kind contributions of computer software and server hosting were provided by the University of Toronto, Masters of Science Program in Biomedical Communications.

Sarah Kim would like to thank Leila and Derek, for their generous mentorship; her committee members, for their guidance; the BMC faculty, for challenging and encouraging her; her classmates, for inspiring her with their workmanship; her family and friends, for their support of all kinds; and Alex, for reminding her that there is more to life than schoolwork. 


\section{References}

American Psychiatric Association. 2000. Diagnostic and statistical manual of mental disorders: DSM-IV-TR. Washington, DC: American Psychiatric Association.

American Psychiatric Association and the Academy of Psychosomatic Medicine. 2015. Dissemination of integrated care within adult primary care settings. Report. 2015.

Andrews, G., Cuijpers, P, Craske, M.G., McEvoy, P., and Titov, N. 2010. Computer therapy for the anxiety and depressive disorders is effective, acceptable and practical health care: a meta-analysis. PLoS One, 5(10): e13196. PubMed https://doi.org/10.1371/journal.pone.0013196

Bereiter, C. 2002. Design Research on Learning Environments. Design Research for Sustained Innovation. Bulletin of the Japanese Cognitive Science Society, 9(3): 321-327.

Collins, A., Joseph, D., and Bielaczyc, K. 2004. Design research: Theoretical and methodological issues. The Journal of the learning sciences, 13(1): 15-42. https://doi.org/10.1207/s15327809j1s1301 2

Gamm, L., Stone S., and Pittman, S. 2010. Mental health and mental disorders-A rural challenge: A literature review. Rural healthy people, 2: 97-113.

Hattie, J. A., and Yates, G.C. 2014. Using feedback to promote learning. Acknowledgments and Dedication, 45.

Heim, C., and Nemeroff, C.B. 2009. Neurobiology of posttraumatic stress disorder. CNS Spectr, 14(1) suppl 1: 13-24. PubMed

Lax, L. 2015. Co-Design of a Pain Interprofessional eLearning Program for Health Sciences Education. Oral Presentation, Association of Medical Illustrators 70th Annual Conference, Cleveland Clinic, Ohio, July 22-25, 2015.

Lax, L. 2011. The PEIR eLearning Design and Usability Survey. The Pain Interprofessional Resource (PEIR) eLearning Program.

https://peir.ca (accessed Dec. 15, 2015)

Lax, L., Taylor, V., Gupta, R., Sockalingam, S., Mason, R. and Chen, Q. 2015. The Online Psychiatric Education Network: Building capacity within communities through eLearning for physicians, health care practitioners and patients. Poster Presentation, Wilson Centre Research Day, Faculty of Medicine, University of Toronto, Toronto, Ontario, Nov. 9, 2015.

Lax, L.R., Russell, M.L., Nelles, L.J., and Smith, C.M. 2009. Scaffolding knowledge building in a Web-based communication and cultural competence program for international medical graduates. Academic Medicine, 84(10): S5-S8. PubMed https://doi.org/10.1097/ACM.0b013e3181b37b4d

Lax, L., Watt-Watson, J., Lui, M., Dubrowski, A., McGillion, M., Hunter, J., MacLennan, C., Knickle, K., Robb, A., and Lapeyre, J. 2011. Innovation and design of a web-based pain education interprofessional resource. Pain Research and Management, 16(6): 427-432. PubMed https://doi.org/10.1155/2011/359079
Mayer, R.E. 2008. Applying the science of learning: evidence-based principles for the design of multimedia instruction. American Psychologist, 63(8): 760. PubMed https://doi.org/10.1037/0003066X.63.8.760

Mayer, R.E. 2014. Research-based principles for designing multimedia instruction. Acknowledgments and Dedication, 59.

Sareen, J. 2015. Posttraumatic stress disorder in adults: impact, comorbidity, risk factors, and treatment. The Canadian Journal of Psychiatry, 59(9): 460-467. PubMed https://doi.org/10.1177/070674371405900902

Sherin, J.E., and Nemeroff, C.B. 2011. Post-traumatic stress disorder: the neurobiological impact of psychological trauma. Dialogues Clin Neurosci, 13(3): 263-278. PubMed

The College of Family Physicians of Canada.2010. Linking Learning to Practice.” Document. From College of Family Physicians Canada, http://www.nationalphysiciansurvey.ca/nps/2010_Survey/Result s/physician1-e.asp (accessed Mar. 15, 2015)

The College of Family Physicians of Canada. 2010. National Physician Survey: 2010 national results by FP/GP or other specialist, sex, age, and all physicians, Section D: Patient access to care. Survey. From National Physician Survey.

http://www.nationalphysiciansurvey.ca/nps/2010_Survey/Result s/physician1-e.asp (accessed Mar. 15, 2015)

The Mental Health Commission of Canada. 2012. Changing Directions, Changing Lives: The Mental Health Strategy for Canada. Ottawa: Mental Health Commission of Canada.

Titov, N., Andrews, G., Davies, M., McIntyre, K., Robinson, E., and Solley, K. 2010. Internet treatment for depression: a randomized controlled trial comparing clinician vs. technician assistance. PloS one, 5(6): e10939. PubMed https://doi.org/10.1371/journal.pone.0010939

Van Ameringen, M., Mancini, C., Patterson, B., and Boyle, M.H. 2008. Post-Traumatic Stress Disorder in Canada. CNS Neuroscience \& Therapeutics, 14(3): 171-181. PubMed https://doi.org/10.1111/j.17555949.2008.00049.x

Wang, P.S., Berglund, P.A., Kessler, R.C., Olfson, M., Pincus, H.A., Wells, K.B. 2005. Failure and delay in initial treatment contact after first onset of mental disorders in the National Comorbidity Survey Replication (NCS-R). Archives of General Psychiatry, 62(6): 603-613. PubMed https://doi.org/10.1001/archpsyc.62.6.603

\section{Authors}

Sarah Kim, BSc, MScBMC. Sarah is a graduate of the Biomedical Communications program, Institute of Medical Sciences, Faculty of Medicine, University of Toronto, Toronto, Canada, who combines her love for science and art to visualize important information in clear and compelling ways. She hopes to not only educate people, but also challenge and engage them to think differently. Sarah enjoyed incorporating her love for hand-lettering into this project and continues to dabble in calligraphy in her free time. She is happily working in Toronto as a Biomedical Communications associate.

Sarah Kim can be contacted atsarah@coactuate.com. 
Leila Lax, BA, BScAAM, MEd, PhD. Leila is an assistant professor in the Master of Science program in Biomedical Communications, Faculty of Medicine, University of Toronto. She teaches medical legal visualization and conducts and supervises design research in health professions education in her Visual Knowledge Building \& Translation Lab. Leila had the pleasure of being Sarah's BMC Supervisor on her Master's Research Project. Leila is the co-Lead on the creation of OPEN.

Dana Ross, MD, MSc, FRCPC. Dana is a psychiatrist in the Trauma Therapy Program at Women's College Hospital. She also lectures in the Department of Psychiatry, Faculty of Medicine, University of Toronto, Canada.

Derek Ng, BSc, MScBMC, PhD. Derek is an assistant professor in the Master of Science program in Biomedical Communications, Faculty of Medicine, University of Toronto, Canada.

Diana Grossi, BASc, MScBMC. Diana is a recent graduate of the Master's program in Biomedical Communications, Faculty of Medicine, University of Toronto, Canada.

\section{Licensing}

The author has chosen to license this content under a Creative Commons Attribution, NonCommercial, NoDerivatives License 4.0 International License.
Renu Gupta, MD, ABPN. Renu is a psychiatrist at Women's College Hospital and a Lecturer in the Department of Psychiatry, Faculty of Medicine, and University of Toronto, Canada.

Sanjeev Sockalingam, MD, MHPE, FRCPC. Sanjeev is the deputy psychiatrist-in-chief at the University Health Network, Toronto General Hospital. He is also the Director of the Continuing Professional and Practice Development at the University of Toronto, Canada.

Robin Mason, PhD. Robin is a scientist in Women's College Research Institute at Women's College Hospital. She is also an assistant professor in the Dalla Lana School of Public Health and Department of Psychiatry at the University of Toronto, Toronto, Canada.

Valerie H. Taylor, MD, PhD, FRCP. Valerie is the Psychiatrist-in Chief at Women's College Hospital and an associate professor in Psychiatry at the University of Toronto., Toronto, Canada. She is also the co-Lead on OPEN. 